\title{
Amantadine for treatment of fatigue in Guillain-Barré syndrome: a randomised, double blind, placebo controlled, crossover trial
}

\author{
M P J Garssen, P I M Schmitz, I S J Merkies, B C Jacobs, F G A van der Meché, P A van Doorn
}

J Neurol Neurosurg Psychiatry 2006;77:61-65. doi: 10.1136/jnnp.2004.046227

See end of article for authors' affiliations

.....................

Correspondence to:

Dr M P J Garssen,

Department of Neurology,

Erasmus Medical Center

Rotterdam, PO Box 1738,

3000 DR Rotterdam, the

Netherlands; m.garssen@

erasmusmc.nl

Received 24 May 2004

In revised form 7 June 2005

Accepted 4 July 2005

\begin{abstract}
Objective: Fatigue is a major complaint in patients with immune mediated polyneuropathies. Despite apparently good physical recovery after Guillain-Barré syndrome (GBS), many patients remain restricted in daily and social activities, and have a decreased quality of life. In this trial, the effect of amantadine on severe fatigue related to GBS was studied.

Methods: During the pre-treatment phase, all patients were monitored for 2 weeks. Only patients with severe fatigue, defined as a mean fatigue score of $\geqslant 5.0$ on the Fatigue Severity Scale (FSS), were randomised for this double blind, placebo controlled, crossover study. Primary outcome measure was improvement of at least 1 point on the FSS. Secondary outcome measures were impact of fatigue, anxiety and depression, handicap, and quality of life.

Results: In total, 80 patients with GBS were randomised, of whom 74 were included for analysis. Fatigue appeared to be reduced already during the pre-treatment phase $(p=0.05)$, probably due to increased attention provided to the patients. No significant differences in any of the primary and secondary outcome measures were found.

Conclusions: Amantadine was not superior to placebo. Because fatigue remains a serious complaint, other studies evaluating new treatment options are strongly recommended.
\end{abstract}

G uillain-Barré syndrome (GBS), an immune mediated disease of the peripheral nervous system, is characterised by acute symmetrical limb weakness and reduction or loss of myotatic reflexes. Sensory deficits and respiratory insufficiency may occur. ${ }^{1}$ Approximately three quarters of patients with GBS experience good neurological recovery after adequate therapy; ${ }^{2}$ however, severe fatigue is a major residual complaint in the majority of patients with immune mediated polyneuropathies. The cause of fatigue is still unknown, but it is significantly associated with a reduced quality of life. ${ }^{3}$ It appears to be independent of muscle strength, sensory deficits, functional ability, and duration of symptoms.

A systematic literature review was conducted evaluating the therapeutic options of fatigue in other immune mediated (neurological) disorders. Treatment options were limited. Besides training intervention studies, ${ }^{4}$ various pharmacological agents as pemoline, modafinil, and amantadine were studied for treating fatigue in multiple sclerosis (MS). ${ }^{5-9}$ Four short term studies demonstrated the efficacy of amantadine in treating fatigue in patients with mild to moderate MS..$^{5-8}$ Amantadine, a NMDA receptor antagonist, blocks presynaptic dopamine reuptake and stimulates postsynaptic receptors. However, its working mechanism in fatigued patients with MS is still poorly understood. To our knowledge, no pharmacological intervention studies aiming to treat fatigue have been performed on patients with GBS to date. Although GBS and MS are certainly not fully comparable, both diseases are immune mediated demyelinating disorders in which relapses may be triggered by infections. ${ }^{10} 11$ Prompted by these observations, we conducted a randomised, double blind, placebo controlled, single centre crossover trial using amantadine, in apparently "well recovered" but severely fatigued patients with GBS.

\section{METHODS}

The study was approved by the ethics committee of Erasmus Medical Center in May 2000. Informed consent was obtained from all participants.

\section{Patients}

In total, 80 neurologically stable patients who had developed GBS and who met the international criteria from the National Institute of Neurological and Communicative Disorders and Stroke ${ }^{1}$ were included in the current study. Patients were recruited from the Dutch GBS databank at the Erasmus Medical Center Rotterdam or the Dutch GBS patients association. Many patients had participated in an earlier study on assessment scales by Merkies et al. ${ }^{3}$

Patients fulfilling the criteria for "severe fatigue", defined as a mean Fatigue Severity Scale score (FSS) of $\geqslant 5.0$, were eligible for inclusion. ${ }^{312}$ A stable neurological clinical condition was defined as no apparent changes in GBS disability score within 3 months before the start of this study, as declared by the patients to their best knowledge. ${ }^{13}$ The onset of GBS was $>6$ months and $<15$ years previously. Patients had to be at least 18 years old, and have a GBS disability score of $\leqslant 3$ (able to walk at least 10 metres with or without aid). ${ }^{13}$

Patients were excluded if they had experienced severe fatigue before developing GBS or if they were suffering from concomitant conditions that might cause fatigue (such as malignancy, chronic infections, anaemia, hypothyroidism, renal and liver disease, chronic fatigue syndrome, human

Abbreviations: CIDP, chronic inflammatory demyelinating polyneuropathy; EHQ, EuroQoL Health Questionnaire; FIS, Fatigue Impact Scale; FSS, Fatigue Severity Scale; GBS, Guillain-Barré syndrome; HAD, Hospital Anxiety and Depression scale; MS, multiple sclerosis; RHS, Rotterdam Handicap Scale; SF-36, Short Form-36 
immunodeficiency virus, chronic obstructive pulmonary disease, cardiovascular disease, diabetes, or other immune mediated disorders). Patients taking medication that could induce fatigue (within 4 weeks before onset of study) were excluded. To avoid a possible confounding effect of depressive symptoms, patients with depression, as defined by a score of more than 10 points on the depression subscale of the Hospital Anxiety and Depression scale (HAD), were excluded. ${ }^{14}$ Pregnancy, breastfeeding, and known contraindications for the use of amantadine, such as renal dysfunction and known allergy, were also exclusion criteria.

Blood values examined were: erythrocyte sedimentation rate, haemoglobin, haematocrit, aspartate transaminase, alanine aminotransferase, $\gamma$-glutamyltransferase, lactic dehydrogenase, alkaline phosphatase, total bilirubin, urea, creatinine, sodium, potassium, glucose, thyroid stimulating hormone, and creatine phosphokinase. If necessary, human chorionic gonadotrophin $\beta$ was evaluated to exclude pregnancy.

\section{Endpoints}

The primary endpoint was reduction of severe fatigue, defined as improvement of at least 1 point on the FSS. ${ }^{312}$ Secondary efficacy variables were changes at the level of impact of fatigue (Fatigue Impact Scale; FIS); ${ }^{15}$ anxiety and depression (Hospital Anxiety and Depression Scale; HAD) $;{ }^{14}{ }^{16}$ handicap (Rotterdam Handicap Scale; RHS) $i^{17}$ and quality of life (Short Form-36; SF-36, ${ }^{18}{ }^{19}$ and EuroQoL Health Questionnaire; EHQ). ${ }^{20}$

\section{Study design}

All patients enrolled in this single centre, randomised, double blind, placebo controlled, $2 \times 2$ crossover trial (fig 1) initially received a letter, enclosing the FSS, requesting their participation. Possible eligible patients visited our outpatient clinic (baseline visit). If a patient met the eligibility criteria, neurological and physical examination (including Medical Research Council sum score, Vigorimeter dynamometry, and GBS disability score) ${ }^{1321}$ was performed, assessment scales were completed, and blood samples were drawn. Only severely fatigued (defined as FFS score $\geqslant 5.0$ ) and nondepressed (defined as HAD depression subscale score $\leqslant 10.0$ ) patients with normal blood values were randomised (at visit 2 ).

\section{Treatment}

To determine the effect on fatigue of extra attention to the patient, one extra visit was scheduled 2 weeks before the

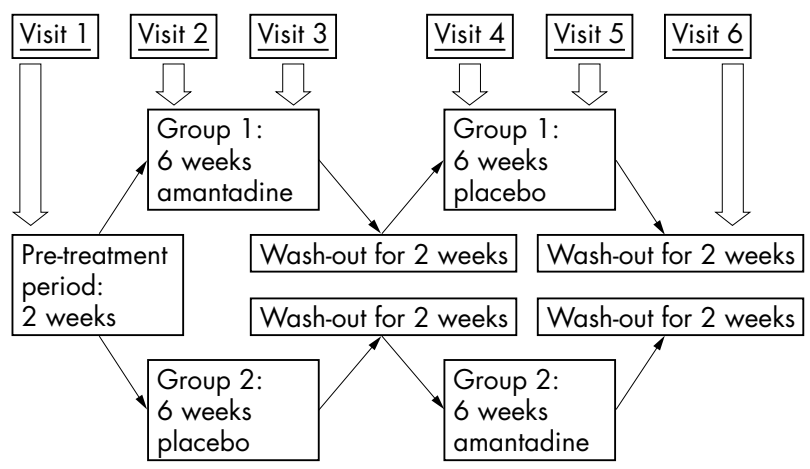

Figure 1 Flow diagram of treatment schedule. Visit 1 (baseline visit) was followed by a pre-treatment period of 2 weeks. At visit 2, patients were randomised and started with the first intervention period, amantadine or placebo. All data were obtained on six consecutive visits; pre-treatment (baseline) visit, start and finish of each intervention period, and post-treatment, 2 weeks after finishing the second intervention period. Each intervention period was followed by a washout period of 2 weeks. start of medication. After this pre-treatment period, patients started the first medication period at follow up visit 2 . Amantadine (100 mg tablets) and placebo tablets indistinguishable in taste, colour, and size were supplied to the investigators by the Department of Pharmacy, Erasmus Medical Center. Patients received amantadine or placebo for 6 weeks. The dosing schedule was one tablet daily taken in the morning during the first week. A second tablet was added in the afternoon in week 2 until week 6 . After a washout period of 2 weeks, patients received the crossover treatment for another 6 weeks. All data were obtained on six follow up visits; pre-treatment, start, and finish of each intervention period, and post-treatment, 2 weeks after finishing the second intervention period (fig 1). Investigators and patients were blinded to treatment group assignment; only the pharmacologist knew the assignments.

\section{Adverse events}

If serious toxicity occurred, the treatment was discontinued and the patient was dropped from the study. Patients were withdrawn if (a) they failed to take the study medication for more than 1 day and/or $(b)$ there were persisting adverse events such as lightheadedness, insomnia, and loss of appetite in combination with nausea for more then 3 consecutive days, hallucinations, convulsions, rash, or ataxia. After each treatment period, the patients were asked whether they had experienced adverse events.

\section{Statistical methods}

Based on data of a small pilot study on the effect of amantadine on fatigue in five patients with GBS and two patients with chronic inflammatory demyelinating polyneuropathy (CIDP) patients, a reduction of 1 point on the FSS seemed clinically relevant. We assumed that $25 \%$ of the patients would improve at least 1 point on the FSS during treatment with placebo, while the percentage of patients improving after amantadine was estimated at $65 \%$ (data not published). Using a two sided alpha of $5 \%$, and a power of $90 \%$, the sample size needed to be be $2 \times 36$, rounded up to $2 \times 40$ patients. ${ }^{22}$ Because fatigue may vary considerably between patients, a crossover design was chosen, to adjust for individual differences.

\section{Randomisation}

The allocation sequence was generated by block randomisation, with a block size of six patients. Each block included three patients starting with amantadine and three starting with placebo, randomly distributed in each block. Before starting this trial, the statistician had sent the allocation sequence list to the pharmacy department. Every consecutive eligible patient seen at the outpatient clinic was given a number from 1 to 80 (assigned in sequence of entering the study). The allocated medication was supplied blinded by an independent pharmacy assistant.

\section{Analyses}

Baseline comparison within treatment groups was performed. The primary endpoint was analysed by comparing paired proportions using the McNemar test. Period and treatment effects were analysed using a one sample $t$ test, and an analysis of variance treating the FSS as a continuous variable. Additional crossover analyses on secondary (continuous) variables were also analysed using analysis of variance. Analysis of the patients' opinion in which period they thought they used amantadine or placebo was performed using Pearson's $\chi^{2}$ test. All calculations were carried out using Stata (version 8.0 for Windows, Stata Statistical Software; Stata Corp., College Station, TX, USA) and Excel (Microsoft Office 2000; Microsoft Corp., USA) software. 


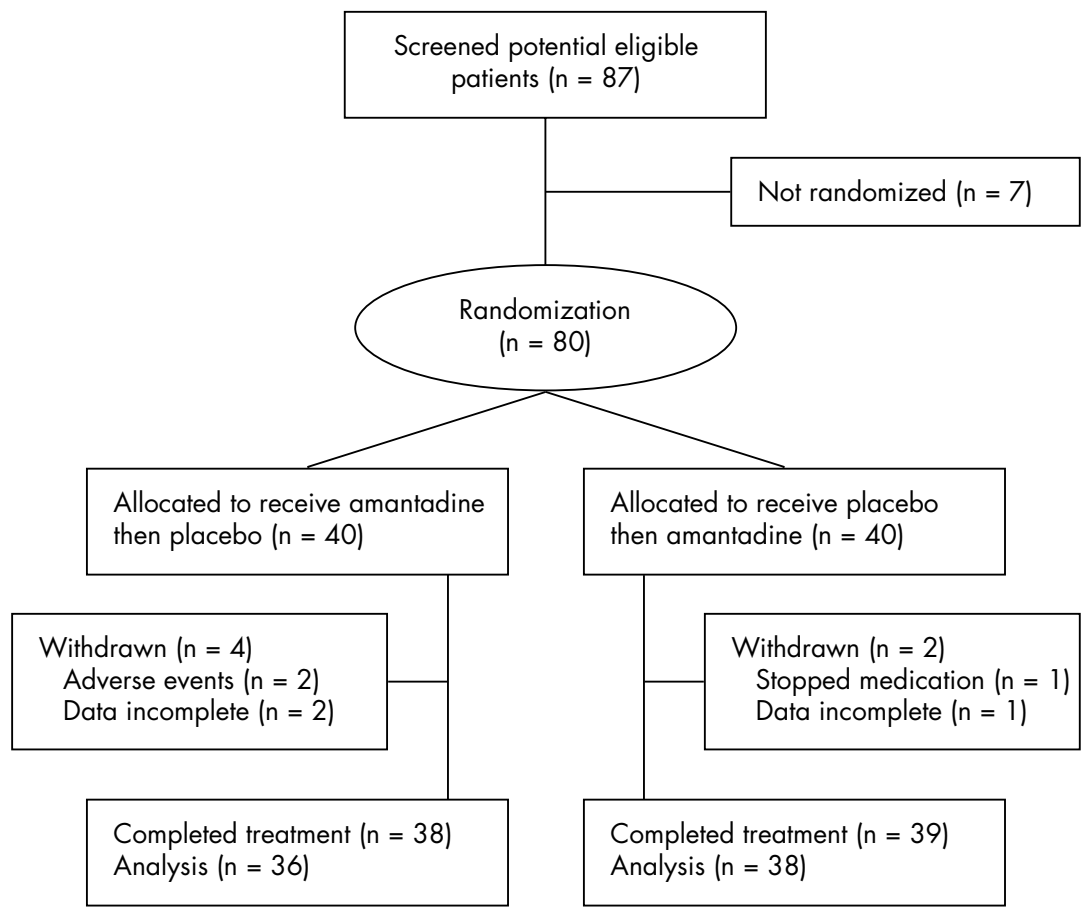

Figure 2 Flow diagram of patient groups.

\section{RESULTS}

\section{Baseline characteristics}

Baseline characteristics are listed in table 1. From 87 potential eligible patients (fig 2), seven patients were not eligible: five patients with an initially eligible FSS score, had a pre-entry score $<5.0$, one patient had severe cardiovascular disease, and the final patient had hypothyroidism, anaemia, and depression. Three randomised patients were withdrawn in the first treatment phase. Two of these were withdrawn while using amantadine; one patient was admitted to another hospital due to an acute cholangitis, while the other patient developed severe complaints of dizziness and strange sensations in his head, persisting for more than three consecutive days. The patient withdrawn from the placebo group thought she might be pregnant and decided to stop immediately. Thus, 77 patients (96\%) completed the trial. As well as the three withdrawn patients, three further patients did not correctly complete all FSS items; visits 2, 3, and 5, respectively. Analysis was therefore performed for 74 patients.

\section{Pre-treatment response}

Based on 74 patients, fatigue reduction almost reached significance when comparing visit 1 with visit $2(p=0.05$, Wilcoxon signed rank test). Despite this pre-treatment decrease in fatigue, fatigue was still sufficiently disabling at visit 2, with a median FSS score of 5.9 in both groups. Seven patients $(9 \%)$ had an FSS score $<5.0$ at the end of this pretreatment phase.

\section{Primary endpoint}

The responses to amantadine and placebo for all individual patients are shown in table 2. From the patients responding to one treatment only, 6 of 11 patients $(55 \%)$ in the group having amantadine first then placebo (amantadine-placebo group) improved after amantadine treatment (odds ratio $(\mathrm{OR})=1.20 ; 95 \%$ confidence interval $(\mathrm{CI}) 0.31$ to 4.97 ; $\mathrm{p}=0.76$, McNemar test). In the placebo-amantadine group, this proportion was 3 of 14 patients $(21 \%)(\mathrm{OR}=0.27 ; 95 \%$

\begin{tabular}{|c|c|c|}
\hline \multirow[b]{2}{*}{ Characteristics } & \multicolumn{2}{|l|}{ Sequence } \\
\hline & $\begin{array}{l}\text { First amantadine, } \\
\text { then placebo }\end{array}$ & $\begin{array}{l}\text { First placebo, } \\
\text { then amantadine }\end{array}$ \\
\hline \multicolumn{3}{|l|}{ Sex, $n$} \\
\hline Female & 19 & 21 \\
\hline Male & 21 & 19 \\
\hline $\begin{array}{l}\text { Median age at start of } \\
\text { study, years (range) }\end{array}$ & $\begin{array}{l}47.5 \\
\text { (19 to } 77)\end{array}$ & $\begin{array}{l}52 \\
(24 \text { to } 82)\end{array}$ \\
\hline $\begin{array}{l}\text { Median duration after } \\
\text { diagnosis years (range) }\end{array}$ & $\begin{array}{l}3.8 \\
10.5 \text { to } 13.11\end{array}$ & $\begin{array}{l}2.6 \\
10.5 \text { to } 15)\end{array}$ \\
\hline \multicolumn{3}{|l|}{ MRC sumscore } \\
\hline $48-57$ & $4(5 \%)$ & $8(10 \%)$ \\
\hline $58-59$ & $12(15 \%)$ & $7(9 \%)$ \\
\hline 60 & $24(30 \%)$ & $25(31 \%)$ \\
\hline \multicolumn{3}{|l|}{ GBS disability score } \\
\hline $\begin{array}{c}\text { distribution, } † \mathrm{n}(\%) \\
]\end{array}$ & $30(38 \%)$ & $26(33 \%)$ \\
\hline 2 & $7(9 \%)$ & $13(16 \%)$ \\
\hline 3 & $3(4 \%)$ & $1(1 \%)$ \\
\hline FSS scoreł, median & 5.9 & 6.0 \\
\hline , & 13.1 to 7.0$)$ & 15.0 to 7.0$)$ \\
\hline \multicolumn{3}{|c|}{$\begin{array}{l}\text { MRC, Medical Research Counsil; GBS, Guillain-Barré syndrome; FSS, } \\
\text { Fatigue Severity Scale. All baseline characteristics were measured at visit } \\
\text { 1. Ranges: *0 to } 60 \text {; †0 to } 6 \text {; } \neq 1 \text { to } 7 \text {. }\end{array}$} \\
\hline
\end{tabular}

CI 0.05 to $1.03 ; \mathrm{p}=0.03$ ), indicating a more favourable outcome for placebo treated patients in this subgroup. However, for both groups of patients combined, this proportion was 9 of 25 patients (36\%) (OR $=0.56$; 95\% CI 0.22 to $1.35 ; \mathrm{p}=0.16)$, indicating no difference between responses after placebo or amantadine. The overall mean difference between the amantadine and placebo period changes in FSS scores was -0.45 (95\% CI -0.94 to 0.04 ; $t=-1.80 ; \mathrm{df}=73 ; \mathrm{p}=0.076)$ which did not quite reach significance, although it favoured amantadine.

To check for order and carryover effects, which could cause bias, we compared the different treatment period responses 
Table 2 Reaching primary endpoint during the different treatment sequences

\begin{tabular}{|c|c|c|c|c|c|c|c|c|c|}
\hline & \multirow{2}{*}{\multicolumn{3}{|c|}{$\begin{array}{l}\text { Amantadine-placebo } \\
\text { group } \\
\geqslant 1 \text { point improvement } \\
\text { during placebo } \\
\text { treatment }\end{array}$}} & \multirow{2}{*}{\multicolumn{3}{|c|}{$\begin{array}{l}\text { Placebo-amantadine } \\
\text { group } \\
\geqslant 1 \text { point improvement } \\
\text { during placebo } \\
\text { treatment }\end{array}$}} & \multirow{2}{*}{\multicolumn{3}{|c|}{$\begin{array}{l}\text { Both groups } \\
\geqslant 1 \text { point improvemen } \\
\text { during placebo } \\
\text { treatment }\end{array}$}} \\
\hline & & & & & & & & & \\
\hline & Yes & No & Total & Yes & No & Total & Yes & No & Total \\
\hline \multicolumn{10}{|c|}{$\begin{array}{l}\geqslant 1 \text { point improvement } \\
\text { during amantadine treatment }\end{array}$} \\
\hline Yes & 2 & 6 & 8 & 1 & 3 & 4 & 3 & 9 & 12 \\
\hline No & 5 & 23 & 28 & 11 & 23 & 34 & 16 & 46 & 62 \\
\hline Total & 7 & 29 & 36 & 12 & 26 & 38 & 19 & 55 & 74 \\
\hline \multicolumn{10}{|c|}{$\begin{array}{l}\text { Reaching the primary endpoint was defined as reduction of at least one point on the FSS. Amantadine-placebo } \\
\text { group shows the changes in period } 1 \text { and } 2 \text { for the group of patients taking amantadine first, then placebo. } \\
\text { Placebo-amantadine group: changes in period } 1 \text { and } 2 \text { for the patients taking placebo, followed by amantadine. } \\
\text { Both groups: changes in period } 1 \text { and } 2 \text { combined for both treatment groups. }\end{array}$} \\
\hline
\end{tabular}

using another method. The mean (SD) improvement in FSS scores in the amantadine-placebo group was $0.46(1.29)$ in the amantadine treatment period and 0.08 (1.44) in the placebo treatment period; for the placebo-amantadine sequence group, scores were -0.03 (1.35) in the amantadine treatment period and $0.4(1.27)$ in the placebo treatment period. The additional two group, $t$ test analysis did not show a significant period effect $(\mathrm{p}=0.078 ; 95 \% \mathrm{CI}-0.92$ to 0.05$)$ or carryover effect ( $\mathrm{p}=0.28 ; 95 \% \mathrm{CI}-1.24$ to 0.44$)$.

\section{Secondary endpoints Fatigue}

No significant differences between the treatment periods within individual patients comparing mean FIS and its subscale scores at the end of treatment period 1 (visit 3 ) and period 2 (visit 5) were observed (one sample $t$ test, $\mathrm{p}=0.77$ ). The FIS showed the same trends in the different treatment periods as were noticed in the FSS.

\section{Anxiety and depression}

Comparing mean HAD scores at the end of treatment periods 1 and 2 did not reveal any difference. There was a slight tendency in favour of the placebo treated group for anxiety $(p=0.06)$ and depression $(p=0.11)$ (one sample $t$ test).

\section{Handicap/quality of life}

The RHS and the EHQ did not show significant differences between the two groups (one sample $t$ test, $\mathrm{p}=0.54$, $\mathrm{p}=0.21)$. No significant differences were seen on comparing mean SF-36 subscale scores in both groups, although the subscales of physical role functioning and mental health perception increased significantly in the placebo treated group ( $t$ test, both $\mathrm{p}=0.008$ ).

\section{Allocation concealment}

If patients used amantadine in allocation period 1, 49\% thought they used active medication in this period, $31 \%$ thought in the second period, and $20 \%$ did not observe any difference. If patients used amantadine in period 2, 44\% thought they used active medication in this treatment period, $44 \%$ thought they used it in the first period, and $12 \%$ had no opinion. No correlation was found in what patients actually used and what they thought they used $(p=0.46)$. By verbal report at the end of the study, patients believed in a slight benefit of "the study drug", without knowing the allocation sequence. Two weeks after the last treatment period, and before unblinding the study, $75 \%$ of patients wanted to continue with open treatment of amantadine compared with placebo.

\section{Adverse effects}

In $40 \%$ of patients, mild and transient side effects were noticed; in 22 patients $(28 \%)$ during amantadine treatment and in 10 patients (13\%) during placebo treatment. Anticholinergic complaints (dry mouth, dizziness) occurred in six patients $(8 \%)$ and were equally divided between both treatment groups. Dizziness, generally mild and transient within 2 or 3 days, was reported directly after starting medication or increasing the dose. Gastrointestinal complaints were noticed in seven patients treated with placebo $(9 \%)$ and in three treated with amantadine $(4 \%)$. The most striking differences were noticed in complaints about sleep; nine patients $(11 \%)$ reported some sleep disturbances when treated with amantadine, and one patient (1\%) while using placebo. These disturbances sometimes persisted for days, although were not sufficiently severe to interrupt trial participation. Other less frequent side effects included headache, feelings of nervousness, and vivid dreams. One patient complained about transient blurred vision directly after starting amantadine.

\section{DISCUSSION}

This is the first study evaluating amantadine as treatment of severe fatigue after GBS. Amantadine was not effective. No significant differences were seen on the FSS between treatment groups. Nevertheless, a certain reduction of fatigue was observed seen both during the pre-treatment period (visit 1 to visit 2), and during the consecutive visits. A possible explanation may be the increased attention given to patients with fatigue during the study and its corresponding ameliorating influence on fatigue. A similar phenomenon was observed in the fatigue treatment studies of Krupp et al. ${ }^{6}$ and the Canadian MS Research Group. ${ }^{7}$ Krupp et al. noticed a decline in fatigue severity for all patients between their first and second study visits, even before starting treatment. ${ }^{6}$ In our study, amantadine did not significantly change levels of anxiety and depression, impact of fatigue, functional disability, handicap scores, and quality of life. Amantadine was generally well tolerated, and side effects were mild and transient (only one patient withdrew because of side effects).

In four short term MS studies it was indicated that fatigue was reduced in $20-40 \%$ of patients using amantadine. The mechanism for the positive response on amantadine in MS is not known. Patients participating in these MS studies had mild to moderate disability, which seemed to be worse than that in the patients with GBS who were mildly affected neurologically in our trial. Despite comparable methods (equivalent dosage of amantadine and duration of treatment periods), differences in underlying pathophysiological mechanisms and in disease course may be explanatory 
factors for the negative effect of amantadine in our study population. In contrast to MS, GBS is usually a monophasic disease without further relapses. Therefore, amantadine should perhaps be studied in patients with a more chronic and still active immune mediated polyneuropathy (for example, CIDP during the long term treatment phase). Regarding the primary outcome measurement, the FSS seemed an adequate questionnaire, as described by Merkies et al. ${ }^{3}$ Although some authors have criticised the FSS for assessing a combination of very general and very specific aspects of the patients' experience of fatigue and for its limited utility in examining the ways in which fatigue affects patients' lives, assessment of fatigue using the FSS and the FIS showed the same results and trends in our study. ${ }^{15} 23$ Both severity and impact of fatigue on cognitive, physical, and social functioning showed corresponding changes on both assessment scales.

Fatigue has been briefly addressed as a residual complaint in a few cases of GBS in the past. ${ }^{24-26}$ It has been noted that physical fatigue often occurred in the first year after onset of GBS, and that fatigue rarely disabled patients. ${ }^{27}$ However, Merkies et al. showed that complaints about fatigue and endurance intolerance may persist for many years.Fatigue, distinguishable from the transient and mild fatigue many healthy persons experience, is still under-recognised by neurologists and rehabilitation physicians. Fatigue remains a severe problem and one of the most important reasons for decrease in quality of life, social life, and physical functioning for years. ${ }^{3}$ Despite studies reporting positive effects of training intervention on fatigue in GBS and CIDP, to date no adequate drug therapy exists. ${ }^{28-31}$

Amantadine did not show a positive effect in this trial, but attention to patients with fatigue seems to be important. Because of the persistence and severity of the complaints, further studies evaluating pathophysiological mechanisms and evaluating other drugs or physical training for "post-GBS fatigue" are indicated.

\section{ACKNOWLEDGEMENTS}

This study was supported by a grant from the Dutch Organization for Scientific Research, NWO (grant no. 940-38-009). The authors would like to thank pharmacist L van Rossum for assistance in preparation and administration of the trial medication.

\section{Authors' affiliations}

M P J Garssen, B C Jacobs, F G A van der Meché, P A van Doorn, Department of Neurology, Erasmus Medical Center Rotterdam, the Netherlands

P I M Schmitz, Department of Statistics, Erasmus Medical Center Rotterdam, the Netherlands

B C Jacobs, Department of Immunology, Erasmus Medical Center Rotterdam, the Netherlands

I S J Merkies, Department of Neurology, Spaarne Hospital, Haarlem, the Netherlands

Competing interests: none

Ethics approval: The study was approved by the ethics committee of Erasmus Medical Center in May 2000. Informed consent was obtained.

\section{REFERENCES}

1 Asbury AK, Cornblath DR. Assessment of current diagnostic criteria for Guillain-Barré syndrome. Ann Neurol 1990;27(suppl):S21-4.
2 Hartung HP, Van Der Meché FG, Pollard JD. Guillain-Barré syndrome, CIDP and other chronic immune-mediated neuropathies. Curr Opin Neurol 1998; 11:497-513.

3 Merkies IS, Schmitz PI, Samiin JP, et al. Fatigue in immune-mediated polyneuropathies. European Inflammatory Neuropathy Cause and Treatment (INCAT) Group. Neurology 1999;53:1648-54.

4 Petajan JH, Gappmaier E, White AT, et al. Impact of aerobic training on fitness and quality of life in multiple sclerosis. Ann Neurol 1996;39:432-41.

5 Murray TJ. Amantadine therapy for fatigue in multiple sclerosis. Can J Neurol Sci 1985; 12:251-4

6 Krupp LB, Coyle PK, Doscher C, et al. Fatigue therapy in multiple sclerosis: results of a double-blind, randomized, parallel trial of amantadine, pemoline, and placebo. Neurology 1995;45:1956-61.

7 The Canadian MS Research Group. A randomized controlled trial of amantadine in fatigue associated with multiple sclerosis. Can J Neurol Sci 1987; 14:273-8.

8 Cohen RA, Fisher M. Amantadine treatment of fatigue associated with multiple sclerosis. Arch Neurol 1989;46:676-80.

9 Rammohan KW, Rosenberg JH, Lynn DJ, et al. Efficacy and safety of modafinil (Provigil) for the treatment of fatigue in multiple sclerosis: a two centre phase 2 study. J Neurol Neurosurg Psychiatry 2002;72:179-83.

10 Giovannoni G, Hartung HP. The immunopathogenesis of multiple sclerosis and Guillain-Barré syndrome. Curr Opin Neurol 1996;9:165-77.

11 Buljevac D, Flach HZ, Hop WC, et al. Prospective study on the relationship between infections and multiple sclerosis exacerbations. Brain 2002; 125:952-60.

12 Krupp LB, LaRocca NG, Muir-Nash J, et al. The fatigue severity scale. Application to patients with multiple sclerosis and systemic lupus erythematosus. Arch Neurol 1989;46:1121-3.

13 Kleyweg RP, van der Meché FG, Schmitz PI. Interobserver agreement in the assessment of muscle strength and functional abilities in Guillain-Barré syndrome. Muscle Nerve 1991;14:1103-9.

14 Zigmond AS, Snaith RP. The hospital anxiety and depression scale. Acta Psychiatr Scand 1983:67:361-70.

15 Fisk JD, Pontefract A, Ritvo PG, et al. The impact of fatigue on patients with multiple sclerosis. Can J Neurol Sci 1994;21:9-14.

16 Spinhoven P, Ormel J, Sloekers PP, et al. A validation study of the Hospital Anxiety and Depression Scale (HADS) in different groups of Dutch subjects. Psychol Med 1997;27:363-70.

17 Merkies IS, Schmitz PI, van der Meché FG, et al. Psychometric evaluation of a new handicap scale in immune-mediated polyneuropathies. Muscle Nerve 2002;25:370-7.

18 Ware JE Jr, Snow KK, Kosinski M, et al. SF-36 health survey. Manual and interpretation guide. Boston: The Health Institute, New England Medical Center, 1997.

19 Aaronson NK, Muller M, Cohen PD, et al. Translation, validation, and norming of the Dutch language version of the SF-36 Health Survey in community and chronic disease populations. J Clin Epidemiol 1998;51:1055-68.

20 The EuroQol Group. EuroQol-a new facility for the measurement of healthrelated quality of life. Health Policy 1990;16:199-208.

21 Merkies IS, Schmitz PI, van der Meché FG, et al. Comparison between impairment and disability scales in immune-mediated polyneuropathies. Muscle Nerve 2003;28:93-100.

22 Pocock SJ. Clinical trials. A practical approach. Chichester: John Wiley \& Sons, 1983.

23 Fisk JD, Ritvo PG, Ross L, et al. Measuring the functional impact of fatigue: initial validation of the fatigue impact scale. Clin Infect Dis 1994;18(suppl 1):S79-S83.

24 Wessely S, Powell R. Fatigue syndromes: a comparison of chronic "postviral" fatigue with neuromuscular and affective disorders. J Neurol Neurosurg Psychiatry 1989;52:940-8.

25 Bernsen RA, de Jager AE, Schmitz Pl, et al. Residual physical outcome and daily living 3 to 6 years after Guillain-Barré syndrome. Neurology 1999;53:409-10.

26 Gregory RJ. Understanding and coping with neurological impairment. Rehabil Nurs 1995;20:74-83.

27 Ropper AH, Wiidicks EFM, Truax BT, et al. Guillain-Barré syndrome. Philadelphia: F A Davis Co, 1991.

28 Pitetti KH, Barrett PJ, Abbas D. Endurance exercise training in Guillain-Barré syndrome. Arch Phys Med Rehabil 1993;74:761-5.

29 Karper WB. Effects of low-intensity aerobic exercise on one subject with chronic-relapsing Guillain-Barré syndrome. Rehabil Nurs 1991;16:96-8.

30 Ruhland JL, Shields RK. The effects of a home exercise program on impairment and health-related quality of life in persons with chronic peripheral neuropathies. Phys Ther 1997;77:1026-39.

31 Garssen MP, Bussmann JB, Schmitz Pl, et al. Physical training and fatigue, fitness, and quality of life in Guillain-Barré syndrome and CIDP. Neurology 2004;63:2393-5. 